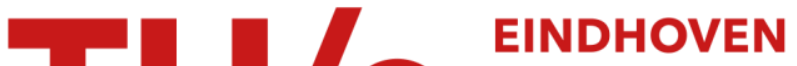 UNIVERSITY OF TECHNOLOGY
}

\section{Tuning the hematite (110) surface properties to enhance its efficiency in photoelectrochemistry}

\section{Citation for published version (APA):}

Podsiadły-Paszkowska, A., Tranca, I., \& Szyja, B. M. (2019). Tuning the hematite (110) surface properties to enhance its efficiency in photoelectrochemistry. Journal of Physical Chemistry C, 123(9), 5401-5410.

https://doi.org/10.1021/acs.jpcc.8b10872

\section{Document license:}

TAVERNE

DOI:

10.1021/acs.jpcc.8b10872

Document status and date:

Published: 07/03/2019

\section{Document Version:}

Publisher's PDF, also known as Version of Record (includes final page, issue and volume numbers)

\section{Please check the document version of this publication:}

- A submitted manuscript is the version of the article upon submission and before peer-review. There can be important differences between the submitted version and the official published version of record. People interested in the research are advised to contact the author for the final version of the publication, or visit the $\mathrm{DOI}$ to the publisher's website.

- The final author version and the galley proof are versions of the publication after peer review.

- The final published version features the final layout of the paper including the volume, issue and page numbers.

Link to publication

\section{General rights}

Copyright and moral rights for the publications made accessible in the public portal are retained by the authors and/or other copyright owners and it is a condition of accessing publications that users recognise and abide by the legal requirements associated with these rights.

- Users may download and print one copy of any publication from the public portal for the purpose of private study or research.

- You may not further distribute the material or use it for any profit-making activity or commercial gain

- You may freely distribute the URL identifying the publication in the public portal.

If the publication is distributed under the terms of Article 25fa of the Dutch Copyright Act, indicated by the "Taverne" license above, please follow below link for the End User Agreement:

www.tue.nl/taverne

Take down policy

If you believe that this document breaches copyright please contact us at:

openaccess@tue.nl

providing details and we will investigate your claim. 


\title{
Tuning the Hematite (110) Surface Properties To Enhance Its Efficiency in Photoelectrochemistry
}

\author{
Agata Podsiadty-Paszkowska, ${ }^{\dagger}$ Ionut Tranca, ${ }^{\dagger}$ and Bartłomiej M. Szyja*,† \\ ${ }^{\dagger}$ Division of Fuels Chemistry and Technology, Faculty of Chemistry, Wrocław University of Science and Technology, Gdańska 7/9, \\ 50-344 Wrocław, Poland \\ ${ }^{\ddagger}$ Department of Mechanical Engineering, Eindhoven University of Technology, P.O. Box 513, 5600MB Eindhoven, The Netherlands
}

Supporting Information

ABSTRACT: We present the analysis of the role of the substitutional doping on the electronic structure of the $\mathrm{Fe}_{2} \mathrm{O}_{3}$ (hematite) (110) surface. The presence of a heteroatom in different crystallographic positions in the surface layer of hematite influences the band structure-additional donor or acceptor states appear in the band gap depending on the type and charge of the heteroatom. The modifications play a role in altering the absorption coefficient, however to a minor extent in the visible-light range. On the other hand, all investigated substitutions seem advantageous for the oxygen evolution

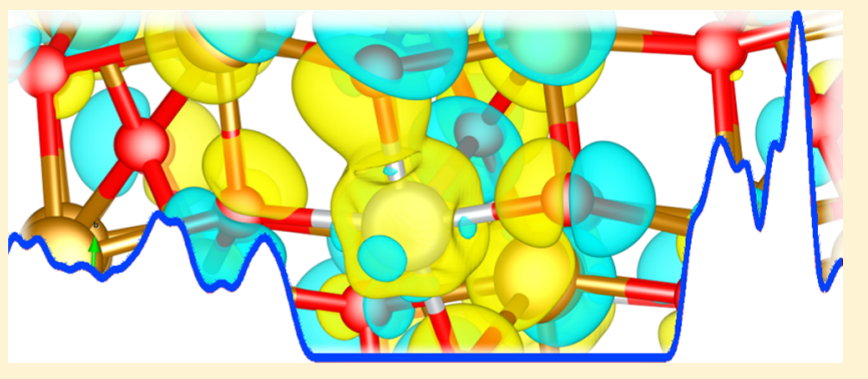
reaction, as for this reaction, the vacuum potential is located inside the band gap. Additionally, the differences in partial charges and binding energy suggest that the substitution site can play a role in preferential binding of the reaction intermediates.

\section{INTRODUCTION}

Photoelectrochemical (PEC) ${ }^{1}$ water-splitting is an elegant solution to the problem of growing energy demand. ${ }^{2,3}$ Regardless if the hydrogen obtained this way is to be used directly as a fuel, or as the reducing agent in chemical processes, the PEC process allows one to utilize a clean energy and minimize the negative impact on the natural environment. In addition to being environmentally friendly, it is a relatively cheap method for hydrogen production.

As water is transparent to visible light, it is not possible to break down its molecules directly; instead, a photocatalyst is required. Commonly used here are the surfaces of the semiconductors that generate required redox potential upon the illumination. The first report of the application of this method was by Fujishima and Honda in $1972 ;{ }^{4}$ it triggered a wide discussion not only on solar energy harvesting and electrocatalytic water-splitting ${ }^{5-7}$ but also on hydrogen storage. 8

$\alpha-\mathrm{Fe}_{2} \mathrm{O}_{3}$ (hematite) is one of the most commonly considered materials to be used as a photoelectrode in PEC hydrogen production. It is cheap, abundant, nontoxic, and chemically stable in aqueous solution of a wide range of $\mathrm{pH}$. It also exhibits a proper width of the band gap: $1.9-2.2 \mathrm{eV},{ }^{9-12}$ making it capable to harvest ca. $30-37 \%$ of solar photons. ${ }^{13}$ Although the width of the band gap of hematite is appropriate for the visible-light range, ${ }^{14}$ the efficiency of this material is limited because of indirect character of this gap. An ideal material should be a direct band gap semiconductor as it makes the optical transition much easier and for that reason more efficient. $^{15}$
Despite intensive research, the hematite-based devices have not yet achieved the theoretically predicted efficiency of 12.7$16.8 \%{ }^{16}$ The main drawback of hematite is its electronic structure with flat bands around the band gap, ${ }^{17}$ which results in very poor conductivity. This means that the electron/hole pairs produced under influence of light recombine quickly. Changing the nature of energy bands and improving conductivity without changing the chemical composition of the material is challenging and requires the use of nanostructures. $^{16,18}$ Unfortunately, investigation of nanostructurization is demanding experimentally as well as computationally. It requires large unit cells to be used in calculations, which drastically increases the demand for computing power and time. The ability to perform such calculations is significantly limited, making nanostructurization fairly unpredictable.

Another important possibility to alter the optical properties is doping of pure oxide material with heteroatoms. ${ }^{19}$ The doping results in direct modification of the electronic structure of the oxide, and depending on the factors such as the ionic radius or oxidation state, can have a significant influence on the resulting redox properties. ${ }^{20}$

Many recent computational studies focus on the bulk hematite properties and doping in the bulk geometry. Arguably, the most important modification of hematite is sulfur doping reported by Xia et al. ${ }^{21}$ They show that sulfur doping significantly lowers the band gap width, and sulfur

Received: November 8, 2018

Revised: February 2, 2019

Published: February 13, 2019 
concentration of $5.6 \%$ improves the properties of the hematite by making a band gap direct. What is more, the modified hematite exhibits the ideal band gap width for solar light harvesting-approximately $1.45 \mathrm{eV}$. Although this result is very promising, it should be noted that doping alone cannot overcome the main problem of hematite, that is, the bands around the energy gap remain flat.

In addition to doping by the anionic sulfur, the doping at the cationic sites has been studied as well. Kosa et al. reported a combined theoretical and experimental study on $\mathrm{Mg}$ doping. ${ }^{22}$ They show that $\mathrm{Mg}$ doping of different concentrations has no significant effect on the band gap, and despite the difference in the oxidation state, the system shows no introduction of holes. They ascribe it to similar effective partial charge on $\mathrm{Mg}$ and $\mathrm{Fe}$ atoms. Huda et al. ${ }^{23}$ tried to modify the hematite band structure by transition-metal $(\mathrm{Sc}, \mathrm{Ti}, \mathrm{Cr}, \mathrm{Mn}$, and $\mathrm{Ni}$ ) doping. They conclude that valence and conduction band edges are modified leading to reduction of the effective mass, but this reduction is negligible. On the other hand, Ti doping of hematite leads to a half-metallic band structure. They also conclude that doping of all the used transition-metal atoms except Sc leads to reduction of the unit cell volume, which affects hopping probability of localized charge carriers.

While this provides an important insight into the efficiency of the process, it needs to be remembered that the PEC processes occur on a semiconductor surface. Thus, the surface effects should be taken into account as they strongly affect the solar energy absorption and oxygen evolution reaction (OER). ${ }^{24,25}$ The most commonly used hematite surface is the (0001) surface of the hexagonal cell, which is known to be stable in different terminations. ${ }^{17,26-29}$

The question of influence of the termination on the efficiency of the OER process is a really nontrivial one, and based on the existing evidence, it can hardly be answered in general terms. Hellman ${ }^{27}$ showed that the stability of the surface with different terminations depends on the applied potential. The major issue is that the terminations that are most stable at zero potential are not stable under potential needed for water-splitting. Ovcharenko ${ }^{29}$ and Huang ${ }^{26}$ show the differences in the free energy for the surfaces with different terminations that affect the properties of the surface, including adhesion, reactivity, and propensity to create defects. Pan ${ }^{19}$ showed that the water molecule is prone to adsorb on the defective site. On the other hand, the defects have an adverse effect on the energy barrier of water-splitting. All those effects described above complicate the selection of the model; however, the detailed investigation of these effects, although intriguing, is beyond the scope of this paper.

In this paper, we propose to use another model, that is, the (110) surface of the rhombohedral cell of hematite, as investigated by the group of Bieberle-Hütter. ${ }^{30}$ The termination of the surface was selected to avoid artificial polarization, as this should make this termination stable, which is in agreement with experimental results. ${ }^{31,32}$ The (110) surface of hematite has not been studied computationally very extensively. ${ }^{30}$ We consider doping this surface with single transition-metal atoms- $\mathrm{Ti}, \mathrm{Co}$, and $\mathrm{Ni}$. These are the elements that have already been used to dope hematite with promising results. ${ }^{33-37}$

It also needs to be stressed out that the model we propose is different from the ones involving bulk doping. Improving the photo-electrocatalytic system performance by bulk doping is relevant for such effects as conductivity and band gap width.
However, catalysis is a surface phenomenon, and surface doping will significantly alter other aspects of photo-electrocatalytic water splitting-for instance, stability of intermediates.

This method is in line with the trend popular in recent years - using dispersed metal atoms as catalytically active sites, that is, single-atom catalysis (SAC). ${ }^{38}$ It has been shown that in many cases, individual metal atoms are active catalysts, while their nanoparticles, although easier to be produced experimentally, are catalytically inactive. ${ }^{39-41}$ Oxides are commonly used as the supports for SAC because of the vacancies and $\mathrm{OH}$ groups present on their surfaces, which are able to stabilize single-metal atoms.

The aim of our research is on the one hand to determine the energy structure of such a hematite surface and the possible effect of doping on improving its optical properties. On the other hand, we want to estimate whether such a surface will be reactive and suitable for the OER study.

\section{MODEL AND COMPUTATIONAL DETAILS}

The calculations were performed within the density functional theory (DFT) framework as implemented in the VASP ${ }^{42,43}$ code. The exchange-correlation energy was used in the Perdew-Burke-Ernzerhof ${ }^{44}$ form. The energy cutoff was set to $500 \mathrm{eV}$ in all calculations. The electron-ion interactions were described by the projector-augmented wave method. ${ }^{43,45}$

The Brillouin zone (BZ) was sampled by $3 \times 2 \times 1 k$-points grid according to the Monkhorst-Pack scheme ${ }^{46}$ for the surface optimization and by $6 \times 4 \times 1 k$-points for the calculation of the electronic properties. The slab consisted of 12 monolayers (MLs) of the $2 \times 2$ unit cell of the hematite (110) surface. Eight of them were fixed at their bulk positions and two MLs on each border of the slab were allowed to move, making the system symmetrical to avoid any possible artificial polarization. The system was relaxed till the forces on each atom were less than $0.03 \mathrm{eV} / \AA ̊$. The bulk calculations for pure and doped hematite were done in a similar geometry, using 4 ML of the $2 \times 2$ (110) surface unit cell. In this calculation, the BZ was sampled by a $3 \times 2 \times 2 k$-points grid for the optimization and $6 \times 4 \times 4 k$-points for the electronic properties calculations.

In order to take into account the strong on-site Coulomb interaction, we used the Hubbard $U$ correction scheme. It is a common practice to fit the $U$ value in a semiempirical way, most often to the experimentally obtained band gap of the investigated material. This is frequently insufficient, as the change of the chemical environment, for instance, upon doping the material, may cause the need to change the $U$ value for the atoms in this environment. Thus, applying the correct values of the $U$ correction is very onerous, as it would even require the use of different values for the same element in different chemical environments. However, having in mind that the differences in the value of the $U$ correction for the atoms of the same element but in different environment are not very significant, it can be assumed that in a similar material, that is, a metal oxide, changes in the description of the electronic state of the system will be minor. In the present work, we have used the following approach to select the $U$ values: for Fe atoms, the $U-J$ value was set to $4.3 \mathrm{eV}$, according to Dudarev's approach, ${ }^{47}$ which was obtained in self-consistent calculations ${ }^{48}$ and is known to reproduce the hematite band gap properly. ${ }^{49}$ For the hematite surface with heteroatoms ( $\mathrm{Ti}, \mathrm{Co}$, or $\mathrm{Ni}$ ) substitutions, we have kept the same $4.3 \mathrm{eV}$ value for 
Fe. For the heteroatoms, we applied the $U-J$ correction values for $\mathrm{Ti}, \mathrm{Co}$, and $\mathrm{Ni}$ as obtained for the oxides of these metals, that is, $10.0 \mathrm{eV},^{50-52} 5.9,,^{53,54}$ and $5.77 \mathrm{eV}^{55}$ respectively.

A $1 \times 1$ surface unit cell contains four nonequivalent $\mathrm{Fe}$ atoms. Two of them differ in the geometrical position at the surface, as they have different $z$-coordinates-they are referred to as the hill and the valley atoms. Both-hill and valley-can also have different magnetic moment directions. We have examined all possible positions of the substituted atoms in this work. The positions of the substitution sites and the frozen layers are shown in Figure 1.

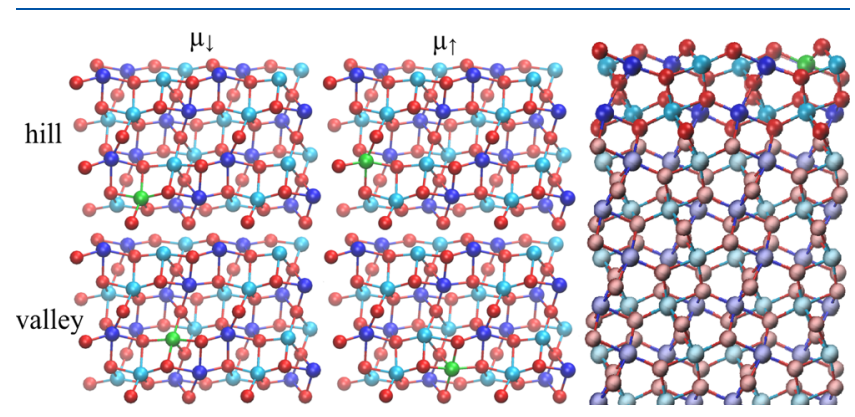

Figure 1. (Left) Four different positions of the atom substitution in the unit cell (top view perspective). (Right) Frozen and relaxed layers in a side view of the surface. Red, blue, and cyan spheres represent oxygen and iron atoms of $\mu_{\uparrow}$ and $\mu_{\downarrow}$, respectively. The substitution site is marked by a green sphere.

Binding energies of the substituted atoms were calculated according to the definition as

$$
E_{\mathrm{b}}=E_{0}+E_{\mathrm{X}}-E_{\text {doped }}
$$

where $E_{0}, E_{\mathrm{X}}$, and $E_{\text {doped }}$ are the total energies of the hematite surface with a single iron vacancy, the single atom of the dopant, and the doped hematite surface, respectively. Partial charges on atoms and bond orders have been calculated using the DDEC6 scheme. ${ }^{56-58}$ We also calculated the real $\left(\epsilon_{1}\right)$ and imaginary $\left(\epsilon_{2}\right)$ parts of the dielectric function to obtain the absorption coefficient using the following equation ${ }^{59}$

$$
\alpha(\omega)=\sqrt{2 \omega} \sqrt{\sqrt{\epsilon_{1}^{2}(\omega)+\epsilon_{2}^{2}(\omega)}-\epsilon_{1}(\omega)}
$$

In order to analyze and visualize the topology of the electron density in conformity with the QTAIM theory, ${ }^{60-62}$ an inhouse written script and freely available AIM-UC software ${ }^{63}$ were used. Bond-critical points (BCPs) were assigned to saddle points along the bond-paths. The Laplacian at a BCP was used to characterize the nature of the pairwise interaction, providing insight into the covalent/ionic character of a bond. Using the electron density, the Laplacian and the virial theorem, the kinetic and potential electronic energy densities were also calculated. The ratio of the potential-energy density $\left|V_{c}\right|$ and the kinetic-energy density $G_{\mathrm{c}}$ at the critical points provides an additional (possibly more sensible) criterion for the identification of the type of the atomic interactions. Values smaller than 1 are characteristic to ionic bonds, while values greater than 2 indicate the covalent bond. Values between 1 and 2 are characteristic for bonds having intermediate ionic/covalent characteristics.

\section{RESULTS AND DISCUSSION}

Surface Model and Binding Energies. Hematite is an antiferromagnet with the ferromagnetic ordering in a (0001) plane and opposite direction of magnetic moments in the consecutive layers. ${ }^{64}$ In our simulations, we have obtained a magnetic moment of the bulk Fe atoms equal to $4.2 \mu_{\mathrm{B}}$, which is about $0.7 \mu_{\mathrm{B}}$ lower than the experimental result. ${ }^{65}$ However, because of the different method used in the present work (different functional used and different $U$ value), we have obtained significantly better results than in the work of Rollmann et al. ${ }^{49}$

A characteristic feature of the (110) surface model is the different $z$-coordinate (perpendicular to the surface) of the $\mathrm{Fe}$ atoms forming the surface. They are further referred to as hill and valley regions. This is in agreement with the previous experimental results and is a characteristic feature of (110) and (012) surfaces of hematite. ${ }^{32}$ It has to be noted that the difference in the position of the hill and valley atoms is relatively small and amounts to $0.1 \AA$. In addition, this effect is only present in the surface layer-in the bulk, the $z$-coordinate of the $\mathrm{Fe}$ atoms are the same.

Despite this small difference in the $z$-coordinate, there are significant differences in the properties of these atoms. Thus, in the pure hematite (110) surface, the binding energy of $\mathrm{Fe}$ atoms amounts to 8.6 and $9.6 \mathrm{eV}$ for the valley and hill, respectively. Additionally, the partial charges of the surface $\mathrm{Fe}$ atoms follow a similar pattern-1.44-1.45 e for the hill, and 1.48-1.49 e for the valley positions. These differences are the result of the surface termination, where the hill $\mathrm{Fe}$ atoms are missing the oxygen in their coordination spheres. This is confirmed by the difference in the sum of bond orders (SBO) of the $\mathrm{Fe}$ atoms, which for all bulk and valley $\mathrm{Fe}$ atoms amounts to 2.62-2.68 and for surface hill atoms to 2.53.

Consequently, similar results can be observed for the heteroatoms substituting different surface $\mathrm{Fe}$ atoms. Table 1

Table 1. Values of Binding Energy (in eV) of a Heteroatom with Different Magnetic Moments and Positions in the Unit Cell

\begin{tabular}{lllll}
\multirow{3}{*}{ hill } & & $\mathrm{Ti}$ & $\mathrm{Co}$ & $\mathrm{Ni}$ \\
& $\mu_{\downarrow}$ & 2.1 & 2.0 & 0.9 \\
valley & $\mu_{\uparrow}$ & 2.1 & 1.8 & 0.9 \\
& $\mu_{\downarrow}$ & 1.4 & 0.7 & 0.6 \\
& $\mu_{\uparrow}$ & 1.4 & 0.6 & 0.6 \\
\hline
\end{tabular}

shows the values of the binding energies of the substituted atoms in each of the tested positions in the surface unit cell. The binding energies do not vary significantly when the heteroatom is located in a position corresponding to the $\mathrm{Fe}$ atom of different magnetic moment site. In fact, for $\mathrm{Ti}$ and $\mathrm{Ni}$, the differences are below the accuracy of the DFT method. The biggest differences are observed for Co and amount to 0.2 and $0.1 \mathrm{eV}$ for the hill and the valley sites, respectively.

On the other hand, the binding energy depends strongly on the position of the heteroatom in the hill or valley location. For each atomic species, the heteroatom in the hill position is bound stronger than in the valley position. This difference is approximately $0.3,0.7$, and $1.3 \mathrm{eV}$ for $\mathrm{Ni}, \mathrm{Ti}$, and $\mathrm{Co}$, respectively. This difference is significant enough to favor the binding of the heteroatoms in the hill position. A similar difference in stability is observed for the nonsubstituted surface, where the difference in binding energy of the Fe atom 
amounts to $0.2 \mathrm{eV}$ for different magnetic moment directions and in the hill/valley position to $1.1 \mathrm{eV}$. This proves that the location of the surface atoms in the hill/valley region contributes more to the binding of a particular atom than the magnetic moment. This observation is consistent with the findings of Noerpel ${ }^{31}$ with respect to binding of $\mathrm{Pb}$ atoms to the $\mathrm{Fe}_{2} \mathrm{O}_{3}(110)$ surface, although the authors attribute the differences in binding to differently coordinated sites.

The low values of binding energy of $\mathrm{Ni}$ atoms to the surface indicate that this kind of doping may be difficult to be obtained experimentally. On the other hand, a different binding energy for the substitutional atom than for $\mathrm{Fe}$ atoms might be beneficial in formation of an active site for the interactions with the water molecules. ${ }^{6-68}$ In fact, all of the investigated atoms bind significantly less strongly to the surface, making all of them promising candidates as co-catalysts in the water-splitting process.

Such a significant reduction in binding energy suggests a change in the bond character; however, the bond orders calculated for the heteroatoms do not fully confirm this. Weak binding of the $\mathrm{Ni}$ atom can be explained by the SBO value higher than the optimal 2 (2.24 in hill and 2.44 in the valley position). A surface atom should have a smaller SBO because of the unsaturated coordination on one side, and the too high SBO suggests that antibonding states are already occupied.

Additionally, for the hill sites, where the binding is stronger, the SBO is lower-which is in agreement with occupation of antibonding states.

The Co-substituted system displaying the biggest differences in binding for hill/valley configurations shows only small differences in the values of SBO. They amount to 2.4 and 2.6 for the valley and hill, respectively, and they are greater than optimal value of 2. However, the Co atoms in the hill configuration are oxidized to +3 [more discussion in the density of states (DOS) section], and this implies that the optimal value of SBO should be 3 . The smaller value obtained is a result of the undercoordination on the surface. Similarly to the $\mathrm{Ni}$ case, the Co in the valley configuration remains on the +2 oxidation state, and as such, the SBO value of 2.4 is too high.

This is supported by the individual bond strength of the Co in the hill configuration-the strongest $\mathrm{Co}-\mathrm{O}$ bond has the order of 0.82 , whereas the $\mathrm{Ni}$ and $\mathrm{Ti}$ bonds with oxygen and the $\mathrm{Co}-\mathrm{O}$ bond in the valley configuration are all of the order of $0.46-0.52$.

The titanium atoms are also characterized by smaller SBO values than $\mathrm{Fe}-2.1$ for hill and only 1.8 for valley sites. This matches the binding energies of Ti heteroatoms because in this case smaller values of the binding energies correspond to the smaller values of SBOs. The values are significantly smaller than the optimal value of 4 , thus, the Ti site forms a potential active site to bind the reactants.

Partial Charges. Figure 2 shows the charge density difference occurring upon substitution of an Fe atom with the heteroatoms. For all the investigated cases, the charge redistribution is observed, but it is mostly of a local character, that is, only the substituted atom and its neighbors are affected.

The smallest and most localized changes are observed for the Co containing system. This holds for both hill and valley sites, despite the difference in binding energy. Both cobalt and nickel are present on the +2 oxidation state, and they bare smaller formal charge than Fe atoms (+3). Partial charges of $\mathrm{Co}$ and of $\mathrm{Ni}$ also are smaller than those of $\mathrm{Fe}$, and for hill and
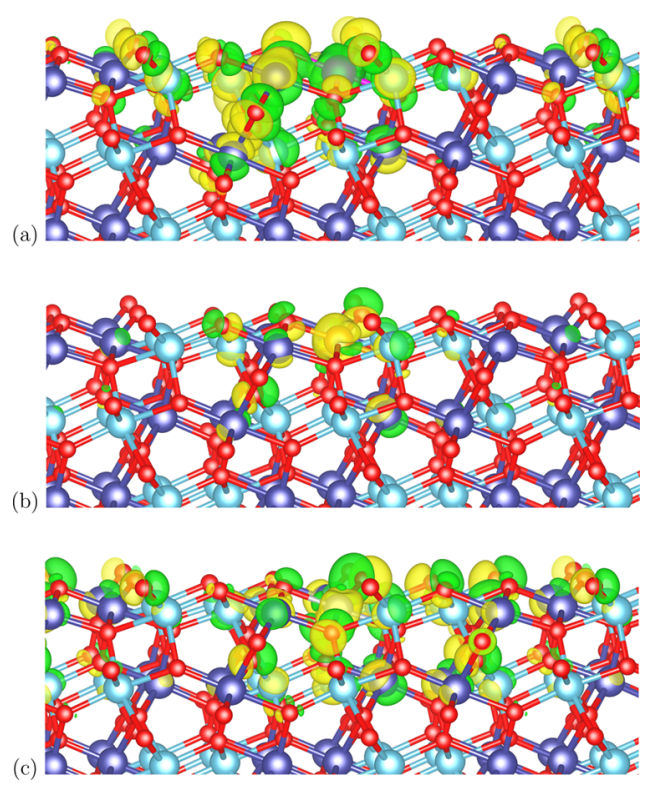

Figure 2. Charge density difference upon substitution of an iron atom with (a) titanium, (b) cobalt, and (c) nickel atoms. Dark blue and light blue spheres represent $\mathrm{Fe}$ atoms with magnetic moment pointing up and down, respectively. Red spheres represent oxygen atoms. Green and yellow colored areas represent accumulation and depletion of the charge, respectively. The isosurfaces are drawn for the 0.02 value.

valley sites, they amount to 1.40 and $1.27(\mathrm{Co})$ and 1.12 and $1.28(\mathrm{Ni})$, respectively. Such a difference in charge is visible in Figure $2 \mathrm{~b}, \mathrm{c}$ as the yellow area at the substitution site. Contrary to that, the titanium atom is present on the +4 oxidation state and bears a partial charge of 2.32 for hill and 2.01 for the valley sites. As the charge on this atom is higher than the one on $\mathrm{Fe}$, it is visible as the green area in the Figure $2 \mathrm{a}$.

Interestingly, besides the charge redistribution affecting merely the closest neighbors, only the repolarization of the charges is observed on the atoms located in the first two layers. This is confirmed by the DDEC6 charges, especially for Co and $\mathrm{Ni}$ systems and the system with $\mathrm{Ti}$ in the valley position, where the difference of the charge on the $\mathrm{Fe}$ atoms is of the order of $0.01 \mathrm{e}$. Contrary to that, a significant charge difference has been observed for the nearest Fe atom in the system with $\mathrm{Ti}$ in the hill site. This is shown in Figure 3a.

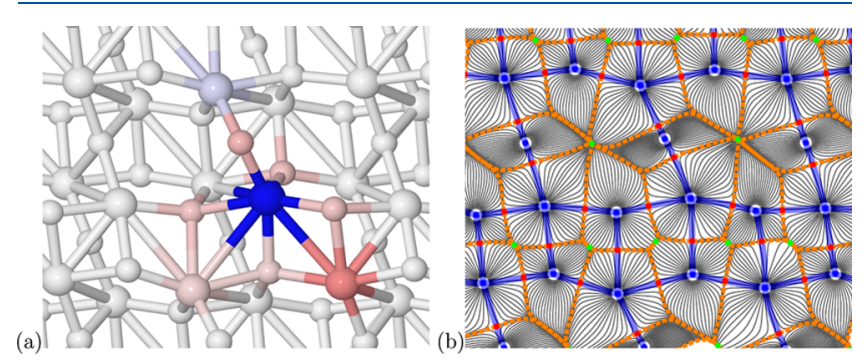

Figure 3. (a) Charge differences caused by the substitution of the hill $\mathrm{Fe}$ atom with the Ti. Colors represent the positive (blue) and negative (red) charge difference with respect to the unmodified $\mathrm{Fe}_{2} \mathrm{O}_{3}$. The darker the shade of blue and red, the more difference in charge is observed. (b) Gradient vector field of electron density for the same system. The red dots denote BCPs. The positions of atoms are matching in both figures. 
The biggest difference is obviously for the Ti heteroatom, which because of the different oxidation state shows significantly higher charge than Fe. The oxygen atoms in the first coordination sphere are slightly affected, but this is also the case for Co- and Ni-substitutions. The most important observation, however, is the change on the $\mathrm{Fe}$ atoms in the second coordination sphere of $\mathrm{Ti}$. The charge on the nearest $\mathrm{Fe}$ atom decreased from 1.44 to $1.10 \mathrm{e}$ and the SBO from 2.53 to 2.25 (the pure $\mathrm{Fe}_{2} \mathrm{O}_{3}$ was used as a reference). This difference is significant enough for the particular $\mathrm{Fe}$ atom to behave differently than other surface $\mathrm{Fe}$ atoms and potentially act as the active site and due to undercoordination to stabilize particular surface intermediates.

The changes in charging of these particular sites are also reflected in slight change of the bonding between a substituted atom and oxygen. Bader analysis classifies bonding interactions into two categories: shared-electron and closed-shell interactions based on the values of electron density and Laplacian at the BCP; however, Cremer and $\mathrm{Kraka}^{69}$ suggested a more detailed descriptor-the ratio of potential-energy density $\left|V_{c}\right|$ and the kinetic-energy density $G_{\mathrm{c}}$ at the critical point. For $\mathrm{Fe}_{2} \mathrm{O}_{3}$, all $\mathrm{Fe}-\mathrm{O}$ bonds are of intermediate character with mostly ionic contribution. This is confirmed by the ratio of potential to kinetic energy at the BCP which is only slightly larger than 1 and varies between 1.071 and 1.275.

For the Ti-system with the substitution in the hill-up position, a slight change in the bonding character has been observed. Two out of five $\mathrm{Ti}-\mathrm{O}$ bonds have the ratio of intermediate value (1.334 and 1.350$)$, which indicates increased covalent character of the bond; however, the covalent contribution is still relatively small. Interestingly, the bonds showing increased covalent character are the ones forming the bridges between the $\mathrm{Ti}$ and those Fe atoms, whose charge changed the most with respect to the unsubstituted surface. This allows us to conclude that the bonding character is responsible for the observed change in charges.

Figure $3 \mathrm{~b}$ shows the atomic basins associated with the $\mathrm{Ti} /$ $\mathrm{Fe}_{2} \mathrm{O}_{3}$ surface layer. The $\mathrm{Fe}, \mathrm{Ti}$, and 2- and 3-coordinated $\mathrm{O}$ atoms can be identified. There is a shift of BCPs on all $\mathrm{Ti}-\mathrm{O}$ bond-paths toward the oxygen atom compared to the $\mathrm{Fe}-\mathrm{O}$ bond, making the basin for Ti atoms larger. This shift is related to bigger electronegativity of $\mathrm{Ti}$ with respect to $\mathrm{Fe}^{70}$ and is not observed for the other two substitutional atoms (Co and $\mathrm{Ni}$ ) as they have almost the same electronegativity as Fe (Figure S7). The biggest shift is observed between the Ti atom and 2coordinated oxygen atom. In addition, the $\mathrm{Fe}$ atom displaying the largest charge difference-shown in the brightest shade of red in Figure 3a-is also characterized by changed path lines in its basin.

Importantly, the cobalt and nickel substitutions, despite the same formal charge, show differences in the charge distribution at the surface. Clearly, the repolarization of the charges is affecting a larger part of the surface for $\mathrm{Ni}$ - than $\mathrm{Co}$-containing system. This is consistent with significant differences in binding energies of $\mathrm{Ni}$ and $\mathrm{Co}$ in the hill sites as well as the difference in partial charges these atoms are bearing ( $1.40 \mathrm{e}$ for $\mathrm{Co}$ and 1.12 e for $\mathrm{Ni}$ ). For the valley substitutions, however, Co and Ni show a similar pattern-a smaller part of the surface is showing the change in charge density. For these two systems, both the binding energies differ by no more than $0.1 \mathrm{eV}$ and the partial charges by 0.09 e. This is a result of the additional doping states overlapping with the surface states, and more detailed explanation will be discussed in the section on the
DOS analysis. A similar pattern is observed for the $\mathrm{Ni}$ substituted in the hill site and all Ti-substituted system, despite clear difference in charge on the subatom described above, and significant difference in binding energy of those atoms.

In all the cases, only the atoms ( $\mathrm{Fe}$ and $\mathrm{O}$ ) from the top two layers displayed the differences in charge density. The atoms located in the subsequent layers did not show any observable differences. This is consistent with the observation of the different electronic states in the surface and in the bulk discussed in the next section.

DOS Analysis. A detailed analysis of the DOS and origin of the peaks observed in the energy gap is complicated by the fact of introduction of the surface and especially its deformation. This causes many nonequivalent atoms in the surface that are located in various chemical environments. This, however, gives only marginally different contributions to DOS. Therefore, we focus on the analysis of the similarities and differences of the DOS in surface and bulk geometries.

Using the $\mathrm{Fe}_{2} \mathrm{O}_{3}(110)$ surface of the rhombohedral cell, we obtain a band gap of approximately $1.48 \mathrm{eV}$, while the gap for bulk geometry in our calculations is $2.10 \mathrm{eV}$ (Figure 4). This
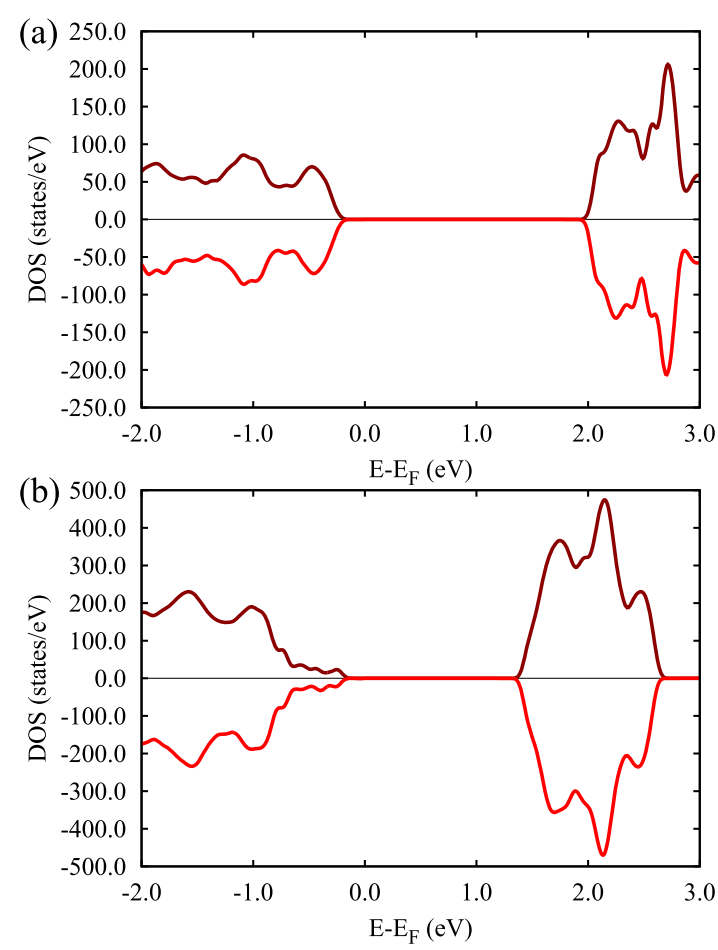

Figure 4. (a) DOS of hematite in bulk geometry. (b) DOS of the (110) hematite surface. Positive and negative values of DOS stand for two different directions of magnetic moment.

narrowing of the band gap is beneficial for the PEC applications, increasing the range of solar energy that can be harvested, but on the other hand, it reduces the redox potential at the surface. Comparing these two values-bulk and surface band gaps-we obtain a difference of approximately $0.6 \mathrm{eV}$. This is a result of additional surface states that are not present in the bulk geometry. ${ }^{71,72}$

Table 2 shows the energy gap values for the doped hematite. The energy gap width does not alter significantly upon doping the hematite surface; thus, in this geometry, the possibility of band gap width modification is limited. This is in contrast to previous findings on narrowing the energy gap in the studies 
Table 2. Values of the Energy Band Gap (in eV) for the Surface Doped with a Subatom with Different Magnetic Moments and Positions in the Unit Cell

\begin{tabular}{|c|c|c|c|c|}
\hline & & $\mathrm{Ti}$ & Co & $\mathrm{Ni}$ \\
\hline \multirow[t]{2}{*}{ hill } & $\mu_{\downarrow}$ & 1.4 & 1.5 & 1.5 \\
\hline & $\mu_{\uparrow}$ & 1.5 & 1.5 & 1.4 \\
\hline \multirow[t]{2}{*}{ valley } & $\mu_{\downarrow}$ & 1.5 & 1.4 & 1.6 \\
\hline & $\mu_{\uparrow}$ & 1.5 & 1.4 & 1.6 \\
\hline
\end{tabular}

using bulk geometry. ${ }^{21-23,73}$ This discrepancy can be explained by the different content of the heteroatoms in those works compared to our model. In the cited papers, in bulk geometry, the content of subatoms ranged from a few to about $10 \%$, while in our calculations, we use one heteroatom atom per 95 Fe atoms. A direct comparison of the content is difficult because of the differences in the computational models-the weight percent of the heteroatoms amounts to $0.6-0.7 \%$, but one needs to remember the thickness of our system is chosen arbitrarily, and depending on the thickness the content of the heteroatoms would be higher or lower. On the other hand, a surface coverage of the heteroatoms would not be possible to calculate for the bulk model.

In almost all the cases, substitution of an $\mathrm{Fe}$ atom leads to the appearance of additional states in the band gap, but these states are of different character. When an $\mathrm{Fe}$ atom is substituted by Ti atom, it leads to the appearance of occupied states (below the Fermi level) in the band gap. This is shown in Figure 5. As Ti has a higher oxidation state $(+4)$ than $\mathrm{Fe}$, it becomes a donor.

On the contrary, $\mathrm{Co}$ and $\mathrm{Ni}$ subatoms show an opposite behavior-both of these species have a lower oxidation state $(+2)$ than $\mathrm{Fe}(+3)$, and their presence in the system leads to the formation of the acceptor, that is, unoccupied states in the band gap-see Figure 5. These states, however, are located close to the Fermi level, and in the case of the Co-substituted system, the Co states are overlapping with the surface states in the valence band. As these states are relatively far from the conduction band, we can conclude that their role in the photoactivity of this material will be limited.

Unexpectedly, in the case of $\mathrm{Ti}$ doping, for the valley position of $\mathrm{Ti}$ and the magnetic moment down, an additional unoccupied state appears in the band gap. A detailed analysis of the DOS shows that the unoccupied state is visible in the spectrum of the atom $\mathrm{Ti}$, but much greater contribution to this state is given by the $\mathrm{Fe}$ atoms of the second layer with the magnetic moment directed down. This effect, however, is too subtle to be clearly seen in the charge density plots. Similarly, in the case of nickel doping in the hill position and the magnetic moment down, an occupied state appears in the spectrum. It is mainly associated with the oxygen atoms of the first layer.

Interestingly, a significant difference is observed for the hill and valley Co substitutions, regardless of the magnetic moment. While the Co atoms in the valley positions are associated with acceptor states, in the hill positions-the Co states overlap with the surface states of the $\mathrm{Fe}_{2} \mathrm{O}_{3}$. These states are below the Fermi level, which means that the Co has changed the oxidation state to +3 when located at the hill site. As Ni does not show such a behavior, we conclude that it stays on its formal oxidation state of +2 . This coincides with the binding energies shown in Table 1 , where Co in the hill position binds more strongly to the surface.
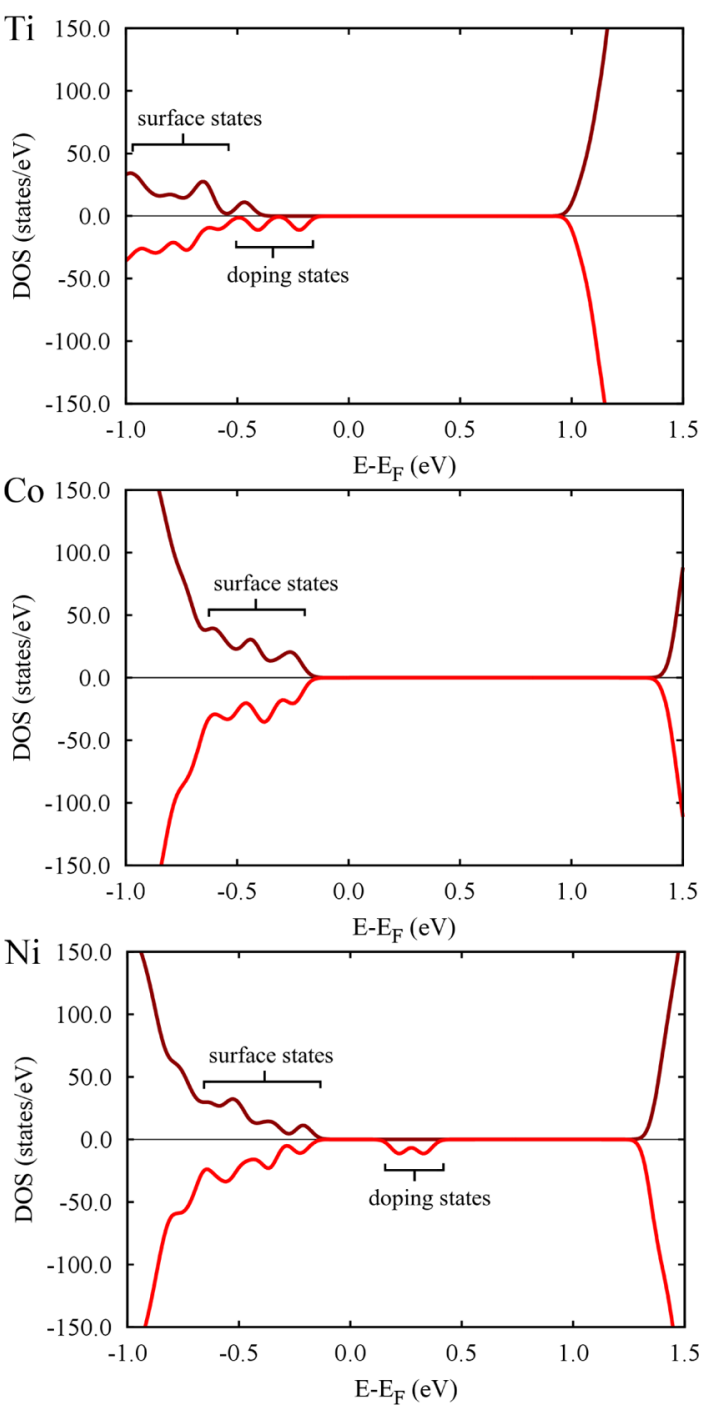

Figure 5. DOS of doped hematite with different substitutional atoms in the hill position with magnetic moment up. Positive and negative values of DOS stand for two different directions of spin.

To make a comparison between bulk and surface calculations, we present densities of states for both casesFigures 5 and 6.

For the bulk material, we have only two possible positions of the substituted atom, that is, with different magnetic moment directions. There is no difference in the $z$-coordinate of particular atoms, contrary to the surface. Using substitutional doping, we obtain just the same value of the band gap as for the pure hematite surface. In each case, it is approximately 2.10 $\mathrm{eV}$; however, considering the positions of the additional states in the band gap, we can expect it to change for the systems with higher content of the dopant, especially $\mathrm{Ti}$.

We also obtain additional donor and acceptor states in the band gap. It indicates clearly that such a small amount of substitutional atoms cannot significantly influence the width of the band gap. The only difference we obtain is the states in the band gap. Because of these states, it is possible to enhance the light absorption, but the small value of their densities suggests that this improvement will not be significant.

Lastly, the possibility of the electron/hole traps formation upon doping needs to be mentioned. Because doping states are always localized, there is always an issue of the trapping. On 

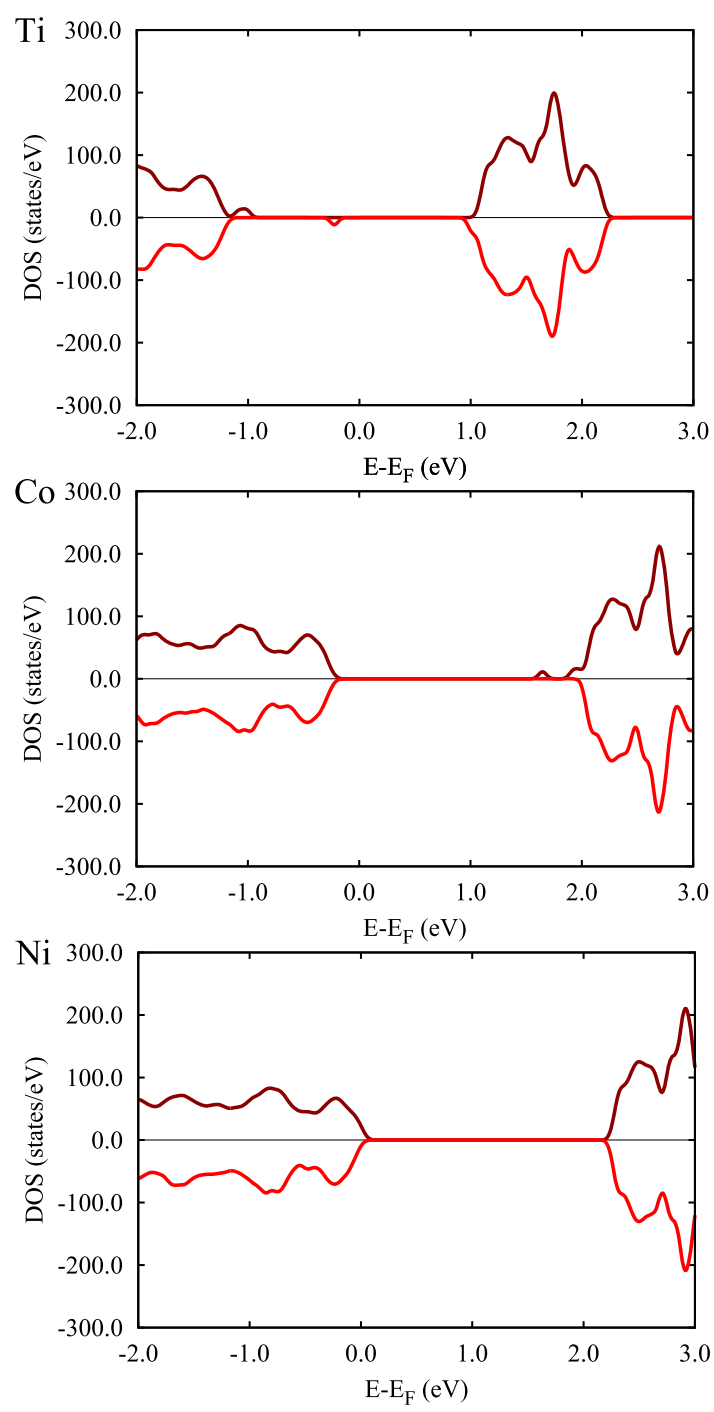

Figure 6. DOS of doped hematite in bulk geometry for one of the magnetic moment direction of a heteroatom. Positive and negative values of DOS stand for two different directions of spin. Because of the symmetry of the system, the densities of states for the heteroatoms magnetic moment of opposite direction are symmetrical.

the other hand, the transition takes place between a localized doping state and a delocalized valence or conduction state. Only if the valence and conduction states were really delocalized could the recombination be restrained. The problem of hematite is flat bands around the band gap, which means quasi-localized states. In our view, the doping cannot significantly influence the rate.

Absorption Coefficient. Figure 7 shows the absorption coefficient versus photon energy for the pure and doped (110) surface of hematite. To facilitate the analysis of the coefficient, the range of visible light was marked on each graph. It is clear that the absorption coefficient increases when atoms of foreign elements are added. This is related not only to the appearance of additional states in the energy gap but also to the increase of DOS around the gap. It can be noticed, however, that the absorption coefficient increases for photon energies higher than $3 \mathrm{eV}$, which are in the range of the UV region. The absorption occurs mainly because of the states around the gap and not in the gap. This is of course beneficial because UV radiation is a part of sunlight; unfortunately, irradiation in this
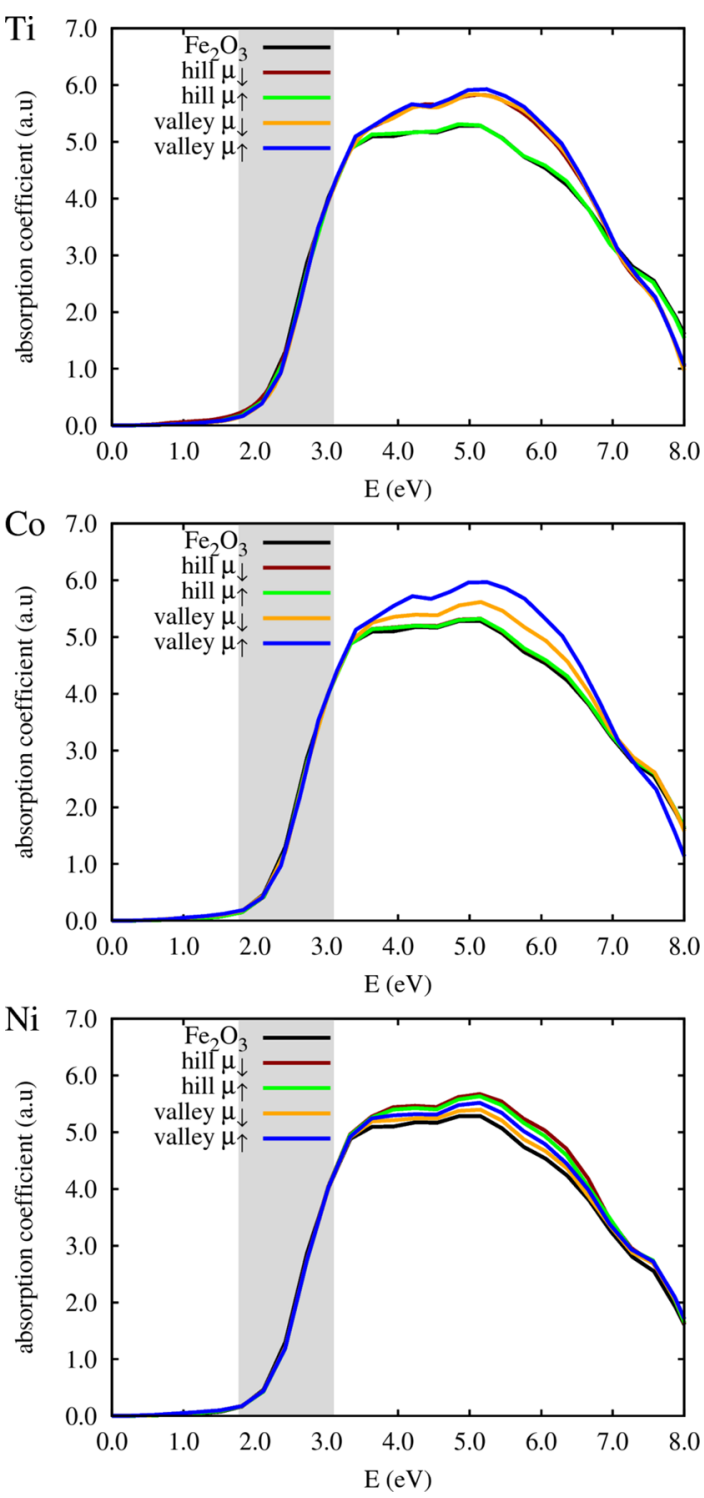

Figure 7. Absorption coefficient of pure and doped hematite surface vs photon energy. Black line labeled as $\mathrm{Fe}_{2} \mathrm{O}_{3}$ shows the results for the pure surface, the other lines are for the doped hematite. The shaded rectangle indicates the visible-light region.

range is much lower than for visible light, which is why such an improvement of the absorption coefficient cannot significantly increase the efficiency of the electron/hole pair formation.

OER Potential. Apart from the absorption study, our goal is to find a proper substrate to study the OER. For this reason, not only the width of the energy gap is important in the research, but also its position relative to the vacuum level. Considering the possibility of water-splitting on different substrates, both potentials need to be taken into account-for hydrogen and oxygen evolution. However, as the focus in this work is the OERs, only this potential is taken into consideration. The surface of hematite was shown to be suited for application in OER in many experimental works. ${ }^{9-12,74-76}$

Table 3 shows the positions of the valence band maximum $(\mathrm{VBM})$ and the conduction band minimum (CBM) related to the vacuum level. For OER, the vacuum potential is -5.67 $\mathrm{eV} .{ }^{77}$ Comparing this value with VBMs and CBMs in the table, it is clear that for each of the substitution, this potential is located inside the energy gap. If we also take into account the 
Table 3. Values of VBM and CBM (in eV) of a Subatom with Different Magnetic Moments and Positions in the Unit Cell Related to the Vacuum Level $0 \mathrm{eV}$

\begin{tabular}{|c|c|c|c|c|c|c|c|}
\hline & & \multicolumn{2}{|c|}{$\mathrm{Ti}$} & \multicolumn{2}{|c|}{ Co } & \multicolumn{2}{|c|}{$\mathrm{Ni}$} \\
\hline & & VBM & CBM & VBM & $\mathrm{CBM}$ & VBM & CBM \\
\hline \multirow[t]{2}{*}{ hill } & $\mu_{\downarrow}$ & -6.93 & -5.52 & -6.47 & -4.98 & -6.53 & -4.99 \\
\hline & $\mu_{\uparrow}$ & -6.40 & -4.91 & -6.49 & -4.97 & -6.55 & -5.12 \\
\hline \multirow[t]{2}{*}{ valley } & $\mu_{\downarrow}$ & -6.41 & -4.95 & -6.64 & -5.21 & -6.69 & -5.13 \\
\hline & $\mu_{\uparrow}$ & -6.28 & -4.81 & -6.62 & -5.21 & -6.62 & -5.02 \\
\hline
\end{tabular}

binding energy considered above, which indicates the possibility of treating the subatoms as the reaction centers, it can be concluded that the use of $\mathrm{Ti}, \mathrm{Co}$, and $\mathrm{Ni}$ as dopants is a promising method for increasing OER efficiency.

\section{CONCLUSIONS}

We have performed a set of DFT calculations for the (110) surface of the rhombohedral cell of hematite aiming at two main goals. The first one was to show a proper method to perform calculations for both doped and undoped materials used for PEC water-splitting and show how the TM doping influences the energetics and optical properties of the hematite surface. The second one was to improve the hematite surface properties as a template for OER.

We have demonstrated the crucial role of using a correct surface geometry to predict the properties of hematite in the context of its applications in water-splitting. Using only the bulk geometry can be one of the problems of water-splitting research, in particular the discrepancy between the theoretical predictions and the experimental results, because it disregards all surface effects. However useful calculations using bulk geometry are (e.g., allowing the determination of the electronic structure in any direction), it should be noted that surface states are critical, and not taking into account surface effects by performing calculations in bulk geometry can lead to erroneous conclusions.

We did not achieve any significant improvement in the optical properties of the surfaces-the substitution leads to the increase of the absorption coefficient, but unfortunately this effect occurs for UV radiation, not for visible light. Similarly, the differences observed for the DOS of doped and clean surfaces are minor. This is due to a low content of the doping sites; however; the position and character of the states coming from the heteroatoms allows us to conclude that significant improvement of the optical character of the material due to doping will be unlikely. This is consistent with the experimental results of Hahn and Mullins, ${ }^{74}$ where the improvement of the absorption coefficient does not depend on the content of the doping $\mathrm{Ti}$.

Most importantly, however, the heterogeneous catalysis is a surface phenomenon, and the doping of the surface atoms will have completely different effect than doping the bulk. So far, the role of a dopant in $\mathrm{Fe}_{2} \mathrm{O}_{3}$ is little understood, especially in terms of its impact on the electronic structure of the material. In our case, the surface was doped with single atoms of $\mathrm{Ti}, \mathrm{Co}$, and $\mathrm{Ni}$. Obtained binding energies indicate that each of the proposed dopants can be a suitable co-catalyst in the watersplitting process on the tested surface; however, the Ni doping is less likely due to the low binding energy, and the incorporation of this species into the surface will probably occur in a different form.
Each of the investigated dopants display a different mechanisms of action that can be beneficial or disadvantageous for the OER. Nickel's weak binding to the surface is the result of the overcoordination in both hill and valley sites, which is confirmed by the SBO to be higher than the optimal value of 2 for $\mathrm{Ni}$ oxides. Besides that, the heteroatom does not impact the properties of the hematite itself. Cobalt display different characteristics in the hill or the valley configurations-even the small difference of the $z$-coordinate can lead to the change in the oxidation state of the Co, which is evidenced by the surface states and states of Co atom located below the Fermi level. Titanium, however, impacts the hematite system the most, where the charge decrease of the nearby $\mathrm{Fe}$ atom suggests its reduction to $\mathrm{Fe}^{+2}$.

\section{ASSOCIATED CONTENT}

\section{Supporting Information}

The Supporting Information is available free of charge on the ACS Publications website at DOI: 10.1021/acs.jpcc.8b10872.

DOS and charge density difference plots of doped hematite with substitutional atoms with different magnetic moments and positions in the unit cell and gradient vector field of electron density of undoped and doped hematite (PDF)

\section{AUTHOR INFORMATION}

\section{Corresponding Author}

*E-mail: b.m.szyja@pwr.edu.pl.

ORCID

Agata Podsiadty-Paszkowska: 0000-0003-3411-8800 Bartłomiej M. Szyja: 0000-0002-0690-9341

Notes

The authors declare no competing financial interest.

\section{ACKNOWLEDGMENTS}

B.M.Sz. and A.P.-P. acknowledge funding from M-ERA.NET Call 2016 for the project "MuMo4PEC" with project number M-ERA.NET 4089. This work is partially financed by National Centre of Science grant no. 2016/23/Z/ST4/04376. The simulations have been performed at the Academic Computing Center TASK in Gdańsk, Poznań Supercomputing and Networking Center and Academic Computer Centre Cyfronet in Kraków. This research was supported in part by PL-Grid Infrastructure.

\section{REFERENCES}

(1) Bard, A. J. Photoelectrochemistry. Science 1980, 207, 139-144.

(2) Ahmad, H.; Kamarudin, S. K.; Minggu, L. J.; Kassim, M. Hydrogen from Photo-Catalytic Water Splitting Process: A Review. Renewable Sustainable Energy Rev. 2015, 43, 599-610.

(3) Jang, J. S.; Kim, H. G.; Lee, J. S. Heterojunction Semiconductors: A Strategy to Develop Efficient Photocatalytic Materials for Visible Light Water Splitting. Catal. Today 2012, 185, 270-277.

(4) Fujishima, A.; Honda, K. Electrochemical Photolysis of Water at a Semiconductor Electrode. Nature 1972, 238, 37-38.

(5) Karlsson, R. K. B.; Cornell, A.; Pettersson, L. G. M. The electrocatalytic properties of doped $\mathrm{TiO}_{2}$. Electrochim. Acta 2015, 180, 514-527.

(6) Szyja, B. M.; van Santen, R. A. Synergy between $\mathrm{TiO}_{2}$ and CoxOy sites in electrocatalytic water decomposition. Phys. Chem. Chem. Phys. 2015, 17, 12486-12491. 
(7) Xu, H.; Zhang, R. Q.; Ng, A. M. C.; Djurišíc, A. B.; Chan, H. T.; Chan, W. K.; Tong, S. Y. Splitting Water on Metal Oxide Surfaces. J. Phys. Chem. C 2011, 115, 19710-19715.

(8) Züttel, A. Hydrogen Storage Methods. Naturwissenschaften 2004, 91, 157-172.

(9) Gilbert, B.; Frandsen, C.; Maxey, E. R.; Sherman, D. M. BandGap Measurements of Bulk and Nanoscale Hematite by Soft X-Ray Spectroscopy. Phys. Rev. B: Condens. Matter Mater. Phys. 2009, 79, 035108.

(10) Akl, A. A. Optical Properties of Crystalline and Non-Crystalline Iron Oxide Thin Films Deposited by Spray Pyrolysis. Appl. Surf. Sci. 2004, 233, 307-319.

(11) Khan, S. U. M.; Akikusa, J. Photoelectrochemical Splitting of Water at Nanocrystallinen- $\mathrm{Fe}_{2} \mathrm{O}_{3}$ Thin-Film Electrodes. J. Phys. Chem. B 1999, 103, 7184-7189.

(12) Glasscock, J. A.; Barnes, P. R. F.; Plumb, I. C.; Bendavid, A.; Martin, P. J. Structural, Optical and Electrical Properties of Undoped Polycrystalline Hematite Thin Films Produced Using Filtered Arc Deposition. Thin Solid Films 2008, 516, 1716-1724.

(13) Hankin, A.; Alexander, J. C.; Kelsall, G. H. Constraints to the Flat Band Potential of Hematite Photo-Electrodes. Phys. Chem. Chem. Phys. 2014, 16, 16176-16186.

(14) Gates, D. M. Spectral Distribution of Solar Radiation at the Earth's Surface. Science 1966, 151, 523-529.

(15) Kittel, C. Introduction to Solid State Physics; John Willey \& Sons, Inc., 1966

(16) Lin, Y.; Yuan, G.; Sheehan, S.; Zhou, S.; Wang, D. HematiteBased Solar Water Splitting: Challenges and Opportunities. Energy Environ. Sci. 2011, 4, 4862-4869.

(17) Pan, H.; Meng, X.; Qin, G. Hydrogen Generation by Water Splitting on Hematite (0001) Surfaces: First-Principles Calculations. Phys. Chem. Chem. Phys. 2014, 16, 25442-25448.

(18) Tilley, S. D.; Cornuz, M.; Sivula, K.; Grätzel, M. Light-Induced Water Splitting with Hematite: Improved Nanostructure and Iridium Oxide Catalysis. Angew. Chem. 2010, 122, 6549-6552.

(19) McFarland, E. W.; Metiu, H. Catalysis by Doped Oxides. Chem. Rev. 2013, 113, 4391-4427.

(20) Ruiz Puigdollers, A.; Schlexer, P.; Tosoni, S.; Pacchioni, G. Increasing Oxide Reducibility: The Role of Metal/Oxide Interfaces in the Formation of Oxygen Vacancies. ACS Catal. 2017, 7, 6493-6513.

(21) Xia, C.; Jia, Y.; Tao, M.; Zhang, Q. Tuning the band gap of hematite $\alpha$-Fe2O3 by sulfur doping. Phys. Lett. A 2013, 377, 19431947.

(22) Kosa, M.; Barad, H. N.; Singh, V.; Keller, D. A.; Shimanovich, K.; Rühle, S.; Anderson, A. Y.; Zaban, A.; Major, D. T. A combined computational and experimental investigation of $\mathrm{Mg}$ doped $\alpha-\mathrm{Fe}_{2} \mathrm{O}_{3}$. Phys. Chem. Chem. Phys. 2016, 18, 781-791.

(23) Huda, M. N.; Walsh, A.; Yan, Y.; Wei, S.-H.; Al-Jassim, M. M. Electronic, structural, and magnetic effects of $3 \mathrm{~d}$ transition metals in hematite. J. Appl. Phys. 2010, 107, 123712.

(24) Yatom, N.; Neufeld, O.; Caspary Toroker, M. Toward Settling the Debate on the Role of Fe2O3 Surface States for Water Splitting. J. Phys. Chem. C 2015, 119, 24789-24795.

(25) Iandolo, B.; Hellman, A. The Role of Surface States in the Oxygen Evolution Reaction on Hematite. Angew. Chem. 2014, 126, $13622-13626$.

(26) Huang, X.; Ramadugu, S. K.; Mason, S. E. Surface-Specific DFT $+\mathrm{U}$ Approach Applied to $\alpha$-Fe2O3(0001). J. Phys. Chem. C 2016, 120, 4919-4930.

(27) Hellman, A.; Pala, R. G. S. First-Principles Study of Photoinduced Water-Splitting on $\mathrm{Fe}_{2} \mathrm{O}_{3}$. J. Phys. Chem. C 2011, 115, 12901-12907.

(28) Souvi, S. M. O.; Badawi, M.; Paul, J.-F.; Cristol, S.; Cantrel, L. A DFT Study of the Hematite Surface State in the Presence of H2, $\mathrm{H} 2 \mathrm{O}$ and O2. Surf. Sci. 2013, 610, 7-15.

(29) Ovcharenko, R.; Voloshina, E.; Sauer, J. Water Adsorption and O-Defect Formation on Fe2O3(0001) Surfaces. Phys. Chem. Chem. Phys. 2016, 18, 25560-25568.
(30) Zhang, X.; Klaver, P.; van Santen, R.; van de Sanden, M. C. M.; Bieberle-Hütter, A. Oxygen Evolution at Hematite Surfaces: The Impact of Structure and Oxygen Vacancies on Lowering the Overpotential. J. Phys. Chem. C 2016, 120, 18201-18208.

(31) Noerpel, M. R.; Lee, S. S.; Lenhart, J. J. X-Ray Analyses of Lead Adsorption on the (001), (110), and (012) Hematite Surfaces. Environ. Sci. Technol. 2016, 50, 12283-12291.

(32) Catalano, J. G.; Fenter, P.; Park, C. Water Ordering and Surface Relaxations at the Hematite (110)-Water Interface. Geochim. Cosmochim. Acta 2009, 73, 2242-2251.

(33) Toussaint, C.; Le Tran, H. L.; Colson, P.; Dewalque, J.; Vertruyen, B.; Gilbert, B.; Nguyen, N. D.; Cloots, R.; Henrist, C. Combining Mesoporosity and Ti-Doping in Hematite Films for Water Splitting. J. Phys. Chem. C 2015, 119, 1642-1650.

(34) Kronawitter, C. X.; Zegkinoglou, I.; Shen, S.-H.; Liao, P.; Cho, I. S.; Zandi, O.; Liu, Y.-S.; Lashgari, K.; Westin, G.; Guo, J.-H.; et al. Titanium Incorporation into Hematite Photoelectrodes: Theoretical Considerations and Experimental Observations. Energy Environ. Sci. 2014, 7, 3100-3121.

(35) Cheng, W.; He, J.; Sun, Z.; Peng, Y.; Yao, T.; Liu, Q.; Jiang, Y.; Hu, F.; Xie, Z.; He, B.; et al. Ni-Doped Overlayer Hematite Nanotube: A Highly Photoactive Architecture for Utilization of Visible Light. J. Phys. Chem. C 2012, 116, 24060-24067.

(36) Saragovi, C.; Arpe, J.; Sileo, E.; Zysler, R.; Sanchez, L. C.; Barrero, C. A. Changes in the structural and magnetic properties of $\mathrm{Ni}$ ? substituted hematite prepared from metal oxinates. Phys. Chem. Miner. 2004, 31, 625-632.

(37) Barrero, C. A.; Arpe, J.; Sileo, E.; Sánchez, L. C.; Zysler, R.; Saragovi, C. Ni- and Zn-Doped Hematite Obtained by Combustion of Mixed Metal Oxinates. Phys. B 2004, 354, 27-34.

(38) Wang, A.; Li, J.; Zhang, T. Heterogeneous Single-Atom Catalysis. Nat. Rev. Chem. 2018, 2, 65-81.

(39) Fu, Q.; Saltsburg, H.; Flytzani-Stephanopoulos, M. Active Nonmetallic $\mathrm{Au}$ and Pt Species on Ceria-Based Water-Gas Shift Catalysts. Science 2003, 301, 935-938.

(40) Zhang, X.; Shi, H.; Xu, B.-Q. Catalysis by Gold: Isolated Surface Au3+ Ions are Active Sites for Selective Hydrogenation of 1,3Butadiene over $\mathrm{Au} / \mathrm{ZrO} 2$ Catalysts. Angew. Chem., Int. Ed. 2005, 44, $7132-7135$

(41) Hackett, S. F. J.; Brydson, R. M.; Gass, M. H.; Harvey, I.; Newman, A. D.; Wilson, K.; Lee, A. F. High-Activity, Single-Site Mesoporous Pd/Al2O3Catalysts for Selective Aerobic Oxidation of Allylic Alcohols. Angew. Chem., Int. Ed. 2007, 46, 8593-8596.

(42) Kresse, G.; Furthmüller, J. Efficient iterative schemes forab initiototal-energy calculations using a plane-wave basis set. Phys. Rev. B: Condens. Matter Mater. Phys. 1996, 54, 11169-11186.

(43) Kresse, G.; Joubert, D. From Ultrasoft Pseudopotentials to the Projector Augmented-Wave Method. Phys. Rev. B: Condens. Matter Mater. Phys. 1999, 59, 1758-1775.

(44) Perdew, J. P.; Burke, K.; Ernzerhof, M. Generalized Gradient Approximation Made Simple. Phys. Rev. Lett. 1996, 77, 3865-3868.

(45) Blöchl, P. E. Projector Augmented-Wave Method. Phys. Rev. B: Condens. Matter Mater. Phys. 1994, 50, 17953-17979.

(46) Monkhorst, H. J.; Pack, J. D. Special Points for Brillouin-Zone Integrations. Phys. Rev. B: Solid State 1976, 13, 5188-5192.

(47) Dudarev, S. L.; Botton, G. A.; Savrasov, S. Y.; Humphreys, C. J.; Sutton, A. P. Electron-Energy-Loss Spectra and the Structural Stability of Nickel Oxide: An LSDA+U Study. Phys. Rev. B: Condens. Matter Mater. Phys. 1998, 57, 1505-1509.

(48) Mosey, N. J.; Liao, P.; Carter, E. A. Rotationally Invariant Ab Initio Evaluation of Coulomb and Exchange Parameters for DFT $+\mathrm{U}$ Calculations. J. Chem. Phys. 2008, 129, 014103.

(49) Rollmann, G.; Rohrbach, A.; Entel, P.; Hafner, J. FirstPrinciples Calculation of the Structure and Magnetic Phases of Hematite. Phys. Rev. B: Condens. Matter Mater. Phys. 2004, 69, 165107.

(50) Arroyo-de Dompablo, M. E.; Morales-García, A.; Taravillo, M. $\mathrm{DFT}+\mathrm{U}$ calculations of crystal lattice, electronic structure, and phase 
stability under pressure of $\mathrm{TiO} 2$ polymorphs. J. Chem. Phys. 2011, 135, 054503.

(51) Deskins, N. A.; Dupuis, M. Electron Transport via Polaron Hopping in Bulk $\mathrm{TiO}_{2}$ : A Density Functional Theory Characterization. Phys. Rev. B: Condens. Matter Mater. Phys. 2007, 75, 195212.

(52) Persson, C.; Ferreira da Silva, A. Strong Polaronic Effects on Rutile TiO2 Electronic Band Edges. Appl. Phys. Lett. 2005, 86, 231912.

(53) Chen, J.; Wu, X.; Selloni, A. Electronic Structure and Bonding Properties of Cobalt Oxide in the Spinel Structure. Phys. Rev. B: Condens. Matter Mater. Phys. 2011, 83, 245204.

(54) Selcuk, S.; Selloni, A. DFT+U Study of the Surface Structure and Stability of Co3O4(110): Dependence on U. J. Phys. Chem. C 2015, 119, 9973-9979.

(55) Floris, A.; de Gironcoli, S.; Gross, E. K. U.; Cococcioni, M. Vibrational Properties of $\mathrm{MnO}$ and $\mathrm{NiO}$ from DFT +U-Based Density Functional Perturbation Theory. Phys. Rev. B: Condens. Matter Mater. Phys. 2011, 84, 161102.

(56) Manz, T. A.; Limas, N. G. Introducing DDEC6 Atomic Population Analysis: Part 1. Charge Partitioning Theory and Methodology. RSC Adv. 2016, 6, 47771-47801.

(57) Limas, N. G.; Manz, T. A. Introducing DDEC6 Atomic Population Analysis: Part 2. Computed Results for a Wide Range of Periodic and Nonperiodic Materials. RSC Adv. 2016, 6, 4572745747.

(58) Manz, T. A. Introducing DDEC6 Atomic Population Analysis: Part 3. Comprehensive Method to Compute Bond Orders. RSC Adv. 2017, 7, 45552-45581.

(59) Fox, M. Optical Properties of Solids; Oxford University Press, 2010.

(60) Henkelman, G.; Arnaldsson, A.; Jónsson, H. A fast and robust algorithm for Bader decomposition of charge density. Comput. Mater. Sci. 2006, 36, 354-360.

(61) Yu, M.; Trinkle, D. R. Accurate and efficient algorithm for Bader charge integration. J. Chem. Phys. 2011, 134, 064111.

(62) Bader, R. Atoms in Molecules: A Quantum Theory; Clarendon Press, 1994.

(63) Vega, D.; Almeida, D. AIM-UC: An application for QTAIM analysis. J. Comput. Methods Sci. Eng. 2014, 14, 131-136.

(64) Sandratskii, L. M.; Uhl, M.; Kübler, J. Band Theory for Electronic and Magnetic Properties of $\alpha-\mathrm{Fe}_{2} \mathrm{O}_{3}$. J. Phys.: Condens. Matter 1996, 8, 983.

(65) Krén, E.; Szabó, P.; Konczos, G. Neutron diffraction studies on the (1-x) $\mathrm{Fe}_{2} \mathrm{O}_{3}-\mathrm{xRh}_{2} \mathrm{O}_{3}$ system. Phys. Lett. 1965, 19, 103-104.

(66) Szyja, B. M.; Vanpoucke, D. Zeolites and Metal-Organic Frameworks from Lab to Industry; Vincent Blay, L. F. B., García, A. C., Eds.; Atlantis Press/Amsterdam University Press, 2018.

(67) Ferrer, S.; Rojo, J. M.; Salmerón, M.; Somorjai, G. A. The Role of Surface Irregularities (Steps, Kinks) and Point Defects on the Chemical Reactivity of Solid Surfaces. Philos. Mag. A 1982, 45, 261269.

(68) Aschauer, U.; He, Y.; Cheng, H.; Li, S.-C.; Diebold, U.; Selloni, A. Influence of Subsurface Defects on the Surface Reactivity of TiO2: Water on Anatase (101). J. Phys. Chem. C 2010, 114, 1278-1284.

(69) Cremer, D.; Kraka, E. Chemical Bonds without Bonding Electron Density ? Does the Difference Electron-Density Analysis Suffice for a Description of the Chemical Bond? Angew. Chem., Int. Ed. Engl. 1984, 23, 627-628.

(70) Kumar, A.; Gadre, S. R. Exploring the Gradient Paths and Zero Flux Surfaces of Molecular Electrostatic Potential. J. Chem. Theory Comput. 2016, 12, 1705-1713.

(71) Tamm, I. Some Remarks on the Theory of Photoelectric Effect on Metals. Phys. Rev. 1932, 39, 170-172.

(72) Shockley, W. Electrons And Holes In Semiconductors; Van Nostrand Reinhold Inc.: U.S., 1950.

(73) Pan, H.; Meng, X.; Liu, D.; Li, S.; Qin, G. (Ti/Zr,N) codoped hematite for enhancing the photoelectrochemical activity of water splitting. Phys. Chem. Chem. Phys. 2015, 17, 22179-22186.
(74) Hahn, N. T.; Mullins, C. B. Photoelectrochemical Performance of Nanostructured Ti- and Sn-Doped $\alpha$-Fe2O3Photoanodes. Chem. Mater. 2010, 22, 6474-6482.

(75) Zhang, X.; Li, H.; Wang, S.; Fan, F.-R. F.; Bard, A. J. Improvement of Hematite as Photocatalyst by Doping with Tantalum. J. Phys. Chem. C 2014, 118, 16842-16850.

(76) Wang, G.; Ling, Y.; Wheeler, D. A.; George, K. E. N.; Horsley, K.; Heske, C.; Zhang, J. Z.; Li, Y. Facile Synthesis of Highly Photoactive $\alpha$-Fe2O3-Based Films for Water Oxidation. Nano Lett. 2011, 11, 3503-3509.

(77) Tamirat, A. G.; Rick, J.; Dubale, A. A.; Su, W.-N.; Hwang, B.-J. Using Hematite for Photoelectrochemical Water Splitting: A Review of Current Progress and Challenges. Nanoscale Horiz. 2016, 1, 243267. 